\title{
Optical properties and morphology of InAs/InP (113)B surface quantum dots
}

\author{
A. Nakkar, ${ }^{1}$ H. Folliot, ${ }^{1, a)}$ A. Le Corre, ${ }^{1}$ F. Doré, ${ }^{1}$ I. Alghoraibi, ${ }^{1}$ C. Labbé, ${ }^{1}$ G. Elias, ${ }^{1}$ \\ S. Loualiche, ${ }^{1}$ M.-E. Pistol, ${ }^{2}$ P. Caroff, ${ }^{2}$ and C. Ellström ${ }^{2}$ \\ ${ }^{1}$ UMR CNRS-6082 FOTON-INSA-Rennes, 20 Avenue des Buttes de Coësmes, CS 14315, \\ F-35043 Rennes Cedex, France \\ ${ }^{2}$ Lund University, Solid State Physics, P.O. Box 118, 22100 Lund, Sweden
}

(Received 31 October 2007; accepted 17 May 2008; published online 12 June 2008)

\begin{abstract}
We report on long-wavelength photoluminescence (PL) emission at room temperature from self-organized InAs surface quantum dots grown by gas-source molecular beam epitaxy on a GaInAsP/InP (113)B substrate. The influence of arsenic pressure conditions during growth on the PL emission of surface quantum dots is detailed as well as oxide/contamination layer formation after growth. Experimental results are in good agreement with six-band $\mathbf{k} \cdot \mathbf{p}$ theory in the envelope function approximation. ㅇ 2008 American Institute of Physics. [DOI: 10.1063/1.2943651]
\end{abstract}

Semiconductor quantum dots (QDs) have attracted considerable interest due to their unique physical properties and application potential. Generally, semiconductor QDs are narrow band gap materials buried in a wide band gap material confining the wave function in all dimensions. However, when located very close to the surface or directly exposed to air, QDs have optical and electronic properties which are very sensitive to fluctuations in the surface potential. ${ }^{1-4}$ Such surface QDs (SQDs) are expected to play an important role for future sensor applications. For example, water-soluble colloidal SQDs have received much attention as biological markers and sensors. ${ }^{5,6}$ Investigation of SQDs is also of interest because of the possibility of establishing a direct correlation between their photoelectronic properties and morphology determined by scanning probe microscopy. ${ }^{7}$

We have investigated surface Stranski-Krastanov InAs QDs grown on substrate InP (113)B by photoluminescence (PL) and atomic force microscopy (AFM). We have compared the emission energy of QDs to calculations and studied the effect of the QD arsenic pressure during growth, which is known to induce strong modifications of the density, shape, and size of InAs/InP QDs. ${ }^{8}$ We have also evidenced the role of a natural oxide/contamination layer affecting both SQDs PL intensity and emission energy.

The studied structures are elaborated by gas-source molecular beam epitaxy (RIBER 32) via Stranski-Krastanov growth mode, that leads to the realization of self-assembled QDs, whose morphology is controlled by the growth conditions. The growth on a high index InP (113)B substrate favors the formation of reduced size InAs QDs, with a high density and a small size dispersion compared to $\operatorname{InP}(001){ }^{9}$ The growth temperature is set at $480{ }^{\circ} \mathrm{C}$ for all samples. The total amount of InAs deposited is 2.1 [(001) equivalent] monolayers, with a growth rate of $0.33 \mathrm{ML} / \mathrm{s}$, on a $\mathrm{Ga}_{0.2} \mathrm{In}_{0.8} \mathrm{As}_{0.435} \mathrm{P}_{0.565}$ quaternary alloy buffer layer emitting at $1.18 \mu \mathrm{m}$ wavelength (referred as Q1.18 hereinafter) and lattice matched to InP. After the QD formation, a $30 \mathrm{~s}$ growth interruption was performed under $\mathrm{As}_{2}$ flow (resulting from cracked $\mathrm{AsH}_{3}$ ) at the same growth temperature. For SQD

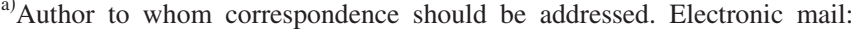
herve.folliot@insa-rennes.fr. samples, a quick cooling to room temperature was performed under $\mathrm{As}_{2}$ flow. The $\mathrm{AsH}_{3}$ flow rate was kept constant during the deposition and growth interruption, with flow rates between 0.3 and 13.4 SCCM (SCCM denotes standard cubic centimeter per minute at STP). Buried QDs (BQDs) were also grown for comparison. For BQDs, the $\mathrm{AsH}_{3}$ growth flow was kept constant (5 SCCM) for all samples and a truncation procedure was used ${ }^{9}$ which allows to accurately control the dot heights from 1.5 up to $3.5 \mathrm{~nm} .^{10}$

SQD samples were then studied by AFM in ambient air. Figure 1 shows AFM images of SQDs for three samples with different $\mathrm{AsH}_{3}$ flow $\left(0.3,5\right.$, and 13.4 SCCM). As the $\mathrm{AsH}_{3}$ flow decreases from 13.4 to $0.3 \mathrm{SCCM}$, the density of the SQDs increases from $5 \times 10^{10}$ to $1 \times 10^{11} \mathrm{~cm}^{-2}$. This increase in density is accompanied by a reduction in the average volume of the SQDs, from 5300 to $1800 \mathrm{~nm}^{3}$. SQDs grown using the lowest $\mathrm{AsH}_{3}$ flow have a reduced average height $(4.8 \pm 1.1 \mathrm{~nm})$ and average diameter $(36 \pm 3 \mathrm{~nm})$ in comparison with SQDs obtained using the highest flow $(7.8 \pm 2.2 \mathrm{~nm})$ and $(48 \pm 4 \mathrm{~nm})$, respectively (uncertainties are one standard deviation). The decrease in the As pressure is thought to be responsible of an increase in the (113)B surface energy and thus favors the nucleation of small islands with a high density. ${ }^{8}$

Next, samples were characterized by continuous PL using a confocal setup at room temperature. The excitation source was a $200 \mathrm{~mW} \mathrm{cw}$ frequency doubled yttrium aluminum garnet laser $(532 \mathrm{~nm})$ that was focused to a $60 \mu \mathrm{m}$ spot, yielding an incident averaged intensity of $4.5 \mathrm{~kW} \mathrm{~cm}^{-2}$. This high intensity was required to get well resolved measure- (a)

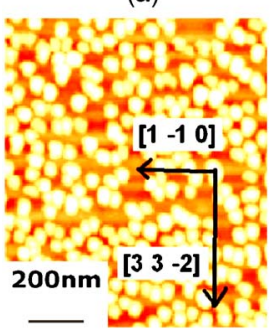

(b)

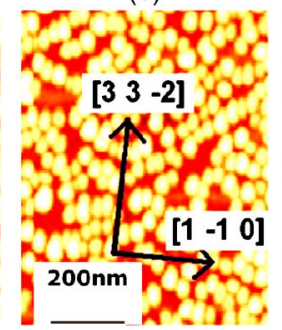

(c)

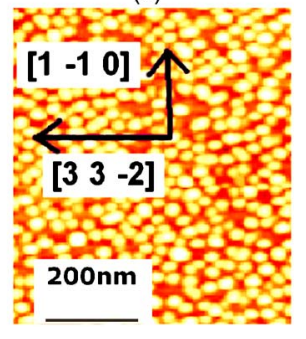

FIG. 1. (Color online) AFM images of SQDs for three samples for different $\mathrm{AsH}_{3}$ flows: (a) 13.4 SCCM, (b) 5 SCCM, and (c) 0.3 SCCM. 


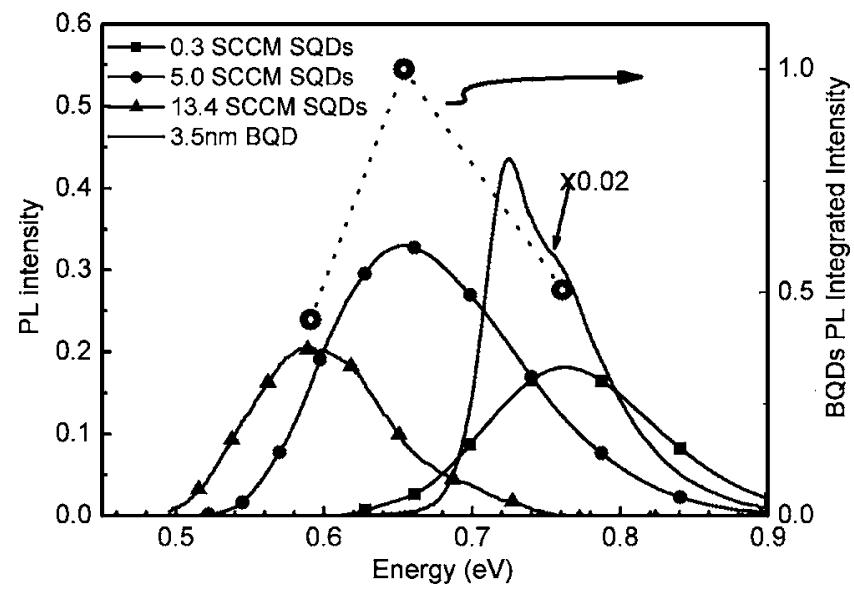

FIG. 2. Room temperature PL spectra for three SQD samples elaborated with three $\mathrm{AsH}_{3}$ flows $(0.3,5$, and 13.4 SCCM) and a BQD sample with a different $3.5 \mathrm{~nm}$ height (left axis) and integrated PL intensity (right axis) for corresponding SQDs.

ments on SQDs due to their rather high surface recombination rate. Sample heating was estimated to be limited to 40-50 K (electron temperature) from high energy bandtail PL analysis on a bulk Q1.18/InP layer, which is consistent with Ref. 11.

The PL was collected back and focused by a $\mathrm{CaF}_{2}$ lens on the entrance slit of a Jobin-Yvon HR1000 spectrometer. The detection of signal is ensured by a double-stage Peltier cooled $\mathrm{PbS}$ photoconductor. Figure 2 shows the nonnormalized PL spectra at room temperature for three samples elaborated with three different $\mathrm{AsH}_{3}$ flows (0.3, 5, and 13.4 SCCM). The emission energy of the biggest SQDs (13.4 $\mathrm{SCCM} \mathrm{AsH}_{3}$ flow) is situated at $0.59 \mathrm{eV}(2.10 \mu \mathrm{m})$, while diminishing $\mathrm{AsH}_{3}$ flow, the peak of PL is shifted toward high energy $0.65 \mathrm{eV}(1.9 \mu \mathrm{m})$ for $5 \mathrm{SCCM}$ and $0.76 \mathrm{eV}(1.63 \mu \mathrm{m})$ for $0.3 \mathrm{SCCM} \mathrm{AsH}_{3}$. For comparison, a PL spectrum of $3.5 \mathrm{~nm}$ high BQDs is also presented. This increase in emission energy observed with the reduction in $\mathrm{AsH}_{3}$ flow is due to an increase in the confinement of the carriers, thanks to a decrease in dimensions. This result is compatible with information obtained by AFM images (Fig. 1). The integrated intensities vary within a factor of about 3 in the whole investigated $\mathrm{AsH}_{3}$ flow range. By comparing the integrated collected PL of SQDs with the buried QDs (BQDs) in Q1.18 (Ref. 9) with an InP capping layer to prevent from nonradiative surface recombination, we note a factor of $\sim 40-70$-fold decrease in the integrated PL intensity which is mainly attributed to surface recombination. Reported carrier lifetime exctracted from time resolved PL of close to the surface $(9 \mathrm{~nm}) \mathrm{InAs} / \mathrm{GaAs}$ QDs have been shown to be in the 65 ps range. ${ }^{12}$ We can expect this value to be even lower for our surface QD. We therefore assume in the following that the carrier density is low and mainly ground QD states contribute to the PL spectra.

The experimental results were then confronted to calculations performed using strain dependent six-band k.p theory in the envelope function approximation. The strain was computed using deformation potential theory. The strain energy was minimized on a grid of $120 \times 120 \times 120$ lattice sites using the finite difference method. ${ }^{13}$ The obtained strain tensor elements were then used as input for the $\mathbf{k}$. p calculation. We did not include the exciton binding energy in the calculations, which we estimate to be about $20 \mathrm{meV}$. Tem-

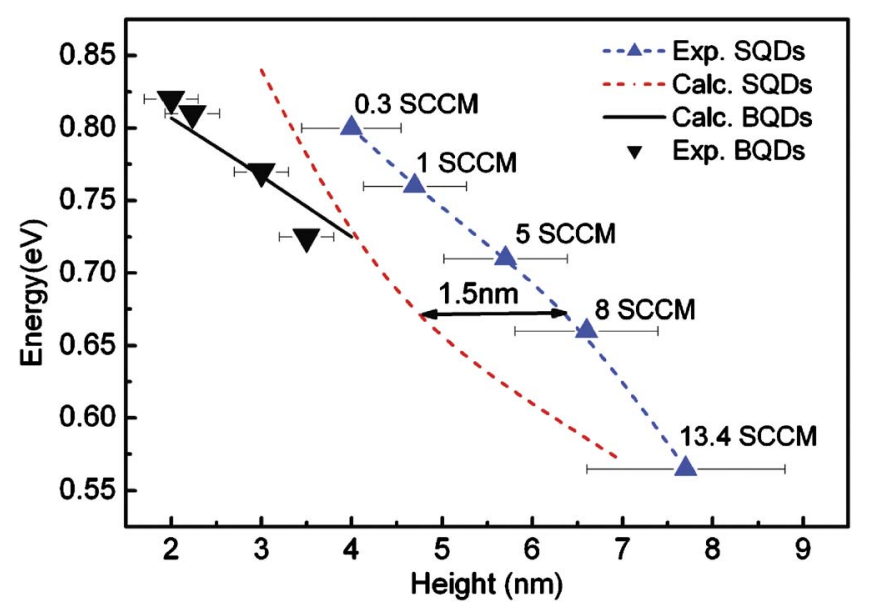

FIG. 3. (Color online) Experimental and calculated room temperature emission wavelengths of InAs SQDs and BQDs on Q1.18/InP (113) B in function their heights. $\mathrm{X}$ error bars are given as one standard deviation from AFM measurements for SQDs and cross-sectional scanning tunneling microscopy measurements for BQDs (from Ref. 10).

perature correction was assumed using the so-called Varshni law for InAs. ${ }^{14}$ The shape of SQDs was assumed to be ellipsoidal, whereas the shape of BQDs was assumed to a truncated ellipsoid as due to the growth truncation procedure.' Figure 3 presents calculated and experimental emission wavelengths of InAs SQDs and InAs BQDs on Q1.18/(113)B InP as a function of dot heights. Indeed, since the SQDs under study keep a rather constant aspect ratio for all the $\mathrm{AsH}_{3}$ flows, the QD PL emission is mainly set by the dot height. ${ }^{9}$ This curve presents a range of emission between 1.55 and $2.18 \mu \mathrm{m}(0.8-0.57 \mathrm{eV})$, for SQDs heights varying from 4 to $7.7 \mathrm{~nm}$ and a range of emission between 1.51 and $1.72 \mu \mathrm{m}$ (0.82 and $0.72 \mathrm{eV}$, respectively) for BQDs with heights varying from 2 to $3.7 \mathrm{~nm}$. The height ranges for both types of QDs (BQDs or SQDs) are different since the effect of capping is known to have a different impact on QD morphology ${ }^{10}$ compared to SQDs which are cooled down under $\mathrm{AsH}_{3}$ flow for AFM observations. Both experimental and calculated curves show a rather parallel tendency. The discrepancy can be attributed to the presence of a thin oxide/ contamination layer with a thickness in the range of $0.7-1.5 \mathrm{~nm}$. This thickness corresponds to the native oxide/ contamination layer on InAs (100) substrate which is reported to be in the $1-2 \mathrm{~nm}$ range. ${ }^{15-17}$ The effect of this oxide/contamination layer is to reduce the AFM observed effective InAs SQD height. ${ }^{18}$ The observed PL spectra half width at one standard deviation (HWSD) at the low energy side in Fig. 2 are 44, 55, and $53 \mathrm{meV}$ for $\mathrm{AsH}_{3}$ flow for 13.4, 5, and 0.3 SCCM SQD, respectively. Calculated HWSD from derivation of the calculated curve on Fig. 3, using the observed standard deviation observed by AFM are 40, 50, and $58 \mathrm{meV}$, respectively, in relatively good agreement with the observed ones. Care must however be taken since the radiative efficiency, which is dependent on the dot height and thus emission energy, ${ }^{19}$ could also affect the spectrum shape.

In order to investigate further the role of the InAs oxide/ contamination layer we performed complementary experiments using AFM and PL. For the low $\mathrm{AsH}_{3}$ sample with $4 \mathrm{~nm}$ AFM height (with highest PL energy emission versus height sensitivity), we conducted surface oxide removal using $2 \% \mathrm{HF}$ in de-ionized water for $30 \mathrm{~s}$. Immediate PL spectrum was then performed, as shown in Fig. 4. This oxide/ 


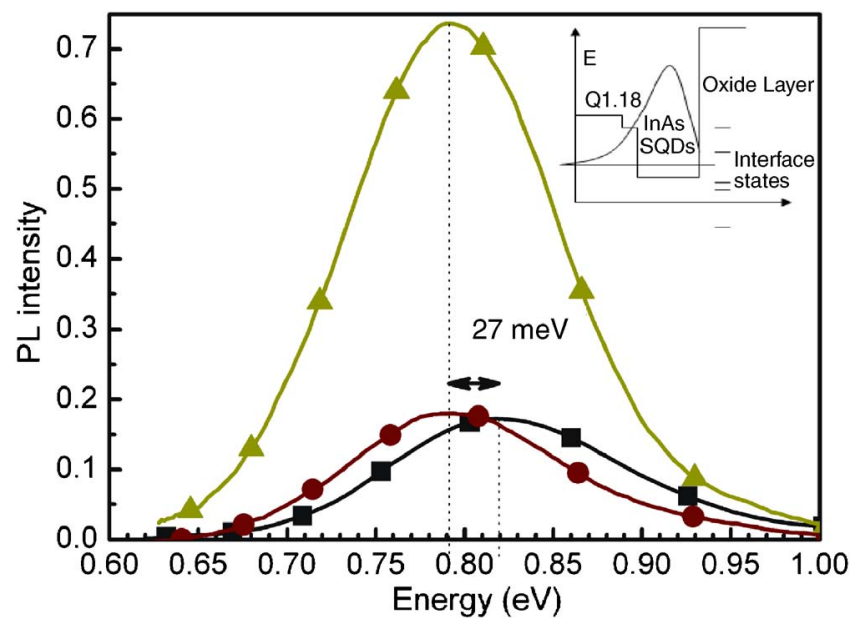

FIG. 4. (Color online) PL spectra recorded on InAs SQDs with $\mathrm{AsH}_{3}$ flow of 0.3 SCCM prior (full circles), immediately after HF $2 \%$ oxide removal (full triangles), and after oxide removal +30 days in air (full squares). The inset shows schematic band diagram with InAs SQDs on Q1.18 with oxide/ contamination covering layer.

contamination layer removal first evidenced an increase in the PL intensity by a factor of 5 , ascribed to a reduced surface recombination velocity, with non-noticeable shift of the PL peak. The oxide layer can be considered as a high energy barrier layer with trap centers, as depicted in Fig. 4 and which almost acts the same as air ${ }^{20,21}$ for the confinement of holes/electrons. The removal of this oxide layer is thus nonnoticeable on the PL spectrum. AFM showed a reduction of the SQDs height of $1.5 \mathrm{~nm}$. Then, the sample was let to oxidize in air for 30 days. AFM measurements first showed an increase in the SQD height of $1.5 \mathrm{~nm}$ corresponding to the oxide/contamination layer. A PL shift of $\sim 27 \mathrm{meV}$ to higher energy was noticed together with a decrease in the PL intensity by the same factor of 5 . By relating this shift to a decrease in the InAs QDs height using our calculations, we find a height reduction of only about $0.2 \mathrm{~nm}$. This value of $0.2 \mathrm{~nm}$ was reported previously on a freshly $\mathrm{HCl}$ etched InAs surface and was ascribed to oxide while a $2 \mathrm{~nm}$ thickness was ascribed to a carbon contaning contamination layer. ${ }^{22}$ These observations are in good agreement with ours. The determination of the precise nature of the oxide/ contamination layer would however be required to fully understand the oxide/contamination effect on the observed PL spectrum.

In summary, we have studied the optical and structural properties of surface InAs/InP (113)B QDs. By controlling the Arsenic flow we modified the morphology of these nano- structures (density, size, homogeneity) and we were able to determine the influence of these parameters on the PL emission wavelength from 1.55 to $2.1 \mu \mathrm{m}$ at room temperature. We have shown the difference in electronic confinement between surface and buried QDs on GaInAsP (Q1.18) and compared our results with the $\mathbf{k} \cdot \mathbf{p}$ theory in the envelope function approximation. The discrepancy between experimental and calculations is interpreted by the presence of a thin $\sim 1.5 \mathrm{~nm}$ InAs oxide/contamination layer.

This work was supported by the SANDIE European Network of Excellence.

The authors thank Assistant Professor Craig Pryor, Department of Physics and Astronomy, University of Iowa, for helpful discussions.

${ }^{1}$ H. Saito, K. Nishi, and S. Sugou, Appl. Phys. Lett. 73, 2742 (1998).

${ }^{2}$ J. Z. Wang, Z. Yang, C. L. Yang, and Z. G. Wang, Appl. Phys. Lett. 77, 2837 (2000).

${ }^{3}$ C. Ellström, J. Trägårdh, L. Samuelson, W. Seifert, M.-E. Pistol, S. Lemeshko, and C. Pryor, Appl. Phys. Lett. 89, 033111 (2006).

${ }^{4}$ B. L. Liang, Zh. M. Wang, and Yu. I. Mazur, Appl. Phys. Lett. 89, 243124 (2006).

${ }^{5}$ W. C. Chen and S. Nie, Science 281, 2016 (1998).

${ }^{6} \mathrm{M}$. Bruchez, P. Morrone, S. Gin, S. Weiss, and A. P. Alivisatos, Science 281, 2013 (1998)

${ }^{7}$ I. A. Karpovich, N. V. Baidus, B. N. Zvonkov, S. V. Morozov, D. O. Filatov, and A. V. Zdoroveishev, Nanotechnology 12, 425 (2001).

${ }^{8}$ P. Caroff, N. Bertru, A. Le Corre, O. Dehaese, T. Rohel, I. Alghoraibi, H. Folliot, and S. Loualiche, Jpn. J. Appl. Phys., Part 2 44, L1069 (2005).

${ }^{9}$ P. Caroff, N. Bertru, C. Platz, O. Dehaese, A. Le Corre, and S. Loualiche, J. Cryst. Growth 273, 357 (2005).

${ }^{10}$ J. M. Ulloaa, P. M. Koenraad, E. Gapihan, A. Létoublon, and N. Bertru, Appl. Phys. Lett. 91, 073106 (2007).

${ }^{11}$ D. Kirillov and J. L. Merz, J. Appl. Phys. 54, 4104 (1983).

${ }^{12}$ C. F. Wang, A. Badolato, I. Wilson-Rae, P. M. Petroff, E. Hu, J. Urayama, and A. Imamoglu, Appl. Phys. Lett. 85, 3423 (2004).

${ }^{13}$ M. Holm, M.-E. Pistol, and C. Pryor, J. Appl. Phys. 92, 932 (2002).

${ }^{14}$ I. Vurgaftman, J. R. Meyer, and L. R. Ram-Mohan, J. Appl. Phys. 89, 5815 (2001).

${ }^{15}$ D. Y. Petrovykh, M. J. Yang, and L. J. Whitman, Surf. Sci. 523, 231 (2003).

${ }^{16}$ G. Hollinger, R. Skheytakabbani, and M. Gendry, Phys. Rev. B 49, 11159 (1994).

${ }^{17}$ I. Shorubalko, A. Pfund, R. Leturcq, M. T. Borgström, F. Gramm, E. Müller, E. Gini, and K. Ensslin, Nanotechnology 18, 044014 (2007).

${ }^{18}$ K. Shiramine, S. Muto, T. Shibayama, N. Sakaguchi, H. Ichinose, T. Kozaki, S. Sato, Y. Nakata, N. Yokoyama, and M. Taniwaki, J. Appl. Phys. 101, 033527 (2007)

${ }^{19}$ Z. Sobiesierski, S. A. Clark, R. H. Williams, A. Tabata, T. Benyattou, G. Guillot, M. Gendry, G. Hollinger, and P. Viktorovitch, Appl. Phys. Lett. 58, 1863 (1991).

${ }^{20}$ J. Dreybrodt, A. Forchel, and J. P. Reithmaier, Phys. Rev. B 48, 14741 (1993).

${ }^{21}$ A. Ismail, J. M. Palau, and L. Lassabatere, J. Appl. Phys. 60, 1730 (1986).

${ }^{22}$ M. Schäfer, W. Naumann, T. Finnberg, M. Hannss, A. Dutschke, and R. Anton, Appl. Surf. Sci. 158, 147 (2000). 